\title{
Trois modalités de réseaux littéraires pour enseigner la grammaire en contexte plurilingue
}

\author{
Joël Thibeault \\ joel.thibeault@uottawa.ca \\ UNIVERSITÉ D'OTTAWA \\ Claude Quevillon Lacasse \\ quevillon_lacasse.claude@uqam.ca \\ UNIVERSITÉ DU QUÉBEC À MONTRÉAL
}

\begin{abstract}
Résumé
La grammaire est une discipline qui est ancrée dans la tradition scolaire francophone et dont l'enseignement, parce qu'on lui reconnait une fonction normative assumée, peut contribuer à la reproduction de certaines inégalités à l'école. Au regard de cet état de fait, le présent article veut, dans un premier temps, mettre en évidence la pertinence didactique que revêtent les réseaux littéraires pour l'enseignement de la grammaire $d u$ français (langue d'enseignement ou seconde) en contexte plurilingue. Ainsi exposerons-nous les fondements théoriques qui concourent à l'utilisation de ces réseaux et verrons-nous comment on peut les mettre en œuvre afin de valoriser et de mettre à profit le répertoire linguistique pluriel des élèves. Dans un second temps, il s'agira d'exemplifier notre propos en présentant trois modalités de réseaux littéraires, ceux-ci, s'ils sont élaborés en considérant les plurilinguismes qui caractérisent de nos jours les salles de classe, pouvant soutenir le développement de la compétence grammaticale des élèves.
\end{abstract}

Mots-clés : grammaire, enseignement, réseaux littéraires, plurilinguisme, écriture

\begin{abstract}
Grammar is a discipline that is anchored in francophone school traditions, and its teaching, because it is associated with a well-known normative function, often contributes to the reproduction of social inequalities at school. In light of this, this article aims, on the one hand, to show the didactic relevance of grammatical literary networks for the teaching of French grammar (whether it be French as a second language or as language of schooling) in plurilingual contexts. We will thus expose the theoretical foundations for the use of these networks and we will see how
\end{abstract}


they can be utilized to enhance the plural linguistic repertoire of students. On the other hand, we will showcase three modalities that these networks can adopt in class and that, when taking into consideration the plurilingual reality of today's classrooms, can support the development of students' grammatical competency.

Key words: grammar, teaching, literary networks, plurilingualism, writing

\section{L'enseignement grammatical par l'entremise de la littérature de jeunesse}

L'enseignement grammatical, sacralisé dans la tradition scolaire francophone (Chervel, 2006), relève encore souvent d'un modèle didactique peu fonctionnel (Vargas, 1996), en ce sens qu'il ne s'articule que rarement à la complexité du vécu linguistique de l'élève et qu'il ne tient que peu compte de ses besoins linguistiques, discursifs et communicatifs. Dans cette perspective, en langue d'enseignement (Lord, 2012) comme en langue seconde (Jean et Simard, 2011), les pratiques traditionnelles d'enseignement grammatical, centrées sur l'exposition et l'exercisation, ont cours dans une proportion importante, malgré les avancées de la recherche dans le domaine.

Par ailleurs, étant donné que l'enseignement grammatical revêt habituellement une fonction normative assumée, c'est-à-dire qu'il vise la transmission de savoirs et de savoir-faire liés au français dans sa variété standard (Chartrand, 2012, 2016), il peut de facto présenter un hiatus vis-à-vis du répertoire linguistique pluriel, fréquemment composé de plusieurs langues, que l'élève se construit au fur et à mesure de ses interactions sociales (De Pietro, 2006; Larouche, 2018). Cet enseignement formel des conventions linguistiques, qui plus est, s'opère encore fréquemment en vase clos, un nombre important d'enseignants peinant à le mettre en lien avec des contextes linguistiques authentiques (Larouche, 2018). Pourtant, selon moult didacticiens provenant de contextes variés d'enseignement du français (Blain, Cavanagh et Cammarata, 2018; Chartrand et Boivin, 2004; Katz et Blyth, 2007; Marmy Cusin et Schneuwly, 2013), c'est en partant de textes authentiques et en analysant les choix linguistiques de l'auteur que l'enseignement de la grammaire peut devenir réellement signifiant pour l'apprenant.

Dans la lignée de la recherche en didactique du français qui fait valoir l'importance d'un enseignement contextualisé de la langue, on constate que la littérature de jeunesse, médium auquel on confère une légitimité croissante en milieu éducatif (Smith, 2016), gagne en popularité au sein de la collectivité de chercheurs en didactique de la grammaire, en langue d'enseignement (Lefrançois, Montésinos-Gelet et Anctil, 2016; Lusetti, 2008) et en langue seconde (Adair-Hauck et Donato, 2002). 
Dupin de Saint-André, Montésinos-Gelet et Bourdeau (2015), à la suite de Tauveron (2002), proposent quant à elles de travailler la littérature de jeunesse en organisant les œuvres par réseaux pouvant reposer sur différents dénominateurs, soit le thème, l'auteur, l'illustrateur, un personnage stéréotype, le genre littéraire, les liens hypertextuels ou intertextuels, et le procédé d'écriture. Ce dernier type de réseau, autour d'un procédé d'écriture, implique le choix d'œuvres partageant un procédé d'écriture commun, par exemple la narration au je. Des activités complémentaires d'observation, d'analyse et d'écriture à partir de ces modèles sont ensuite proposées aux élèves afin que, à partir d'un contexte authentique, ils s'approprient le procédé d'écriture ciblé. Or, il nous est apparu que des éléments grammaticaux, comme le choix des temps verbaux, la répétition de structures syntaxiques particulières, la mise en évidence de groupes syntaxiques et l'énumération de compléments, pouvaient également servir de dénominateur commun pour l'élaboration d'un réseau littéraire. Ainsi, depuis maintenant quelques années, les travaux que nous menons (Thibeault et Quevillon Lacasse, 2016a, 2016b) proposent le décloisonnement de l'enseignement grammatical, dès lors arrimé à l'exploration d'œuvres pour la jeunesse organisées en réseaux littéraires. Nous avons conceptualisé ce que nous nommons le réseau littéraire grammatical (RLG), que nous définissons comme étant un regroupement d'œuvres pour la jeunesse qui permettent l'exploration de concepts grammaticaux similaires ou complémentaires. Nous avons en outre identifié cinq raisons qui justifient la démarche didactique que nous suggérons :

\section{Le RLG permet d'enseigner la grammaire à partir de l'observation de phénomènes.}

Les didacticiens de la grammaire, à la lumière des avancées en psychologie cognitive, préconisent un enseignement grammatical principalement inductif (Beaulne et Gauvin, 2017; Chartrand, 1996; Ellis et Shintani, 2014), qui positionne l'apprenant au cœur de son apprentissage, qui l'encourage à observer des corpus et, à l'aide d'instruments de classification favorisant la réflexion linguistique et du soutien de ses pairs, à poser des hypothèses sur le fonctionnement de l'unité linguistique à l'étude. Le recours à la littérature de jeunesse peut dès lors être à la source d'un travail grammatical axé sur la collaboration, le doute et, surtout, la découverte.

2. Le RLG permet d'enseigner un concept grammatical de manière progressive.

En choisissant les œuvres de manière réfléchie, on peut faire observer un concept grammatical dans une perspective de progression, notamment selon la complexité linguistique de structures linguistiques. Par 
exemple, si un auteur fait un usage fréquent des réalisations simples du complément de phrase (p. ex., les groupes nominal et prépositionnel), d'autres auteurs opteront pour des réalisations plus complexes (p. ex., la subordonnée complément de phrase). En réunissant de telles œuvres dans un réseau, on peut engager l'élève dans un apprentissage étayé et, donc, le guider dans une conceptualisation progressive de l'objet étudié (Thibeault et Quevillon Lacasse, 2016b).

3. Le RLG permet aux élèves d'explorer les effets des choix linguistiques de l'auteur sur le lecteur.

Une exploration de phénomènes grammaticaux à partir d'un contexte littéraire permet à l'élève d'observer l'incidence énonciative des choix linguistiques de l'auteur sur le lecteur (Lefrançois, Montésinos-Gelet et Anctil, 2018). La grammaire devient donc un ensemble de ressources qui sont mobilisées par un auteur afin de créer du sens, une vision se rapprochant de la perspective systémique fonctionnelle (Halliday et Matthiessen, 2014).

\section{Le RLG permet de stimuler l'écriture.}

Certaines œuvres, particulièrement celles qui adoptent des structures syntaxiques répétitives, peuvent servir d'inspiration pour des projets d'écriture stimulants. Notons, par exemple, des projets d'écriture à la manière d'un auteur, lors desquels l'élève est appelé à imiter les choix syntaxiques d'un auteur dans sa production écrite (Chénard-Guay, 2011; Hirvela, 2016; Lefrançois, Montésinos-Gelet et Anctil, 2018), et les exercices visant le développement de la fluidité, comme le jogging d'écriture (Montésinos-Gelet, 2016).

\section{Le RLG permet la réflexion sur la (les) langue(s).}

L'utilisation de réseaux littéraires grammaticaux permet in fine le développement d'une réflexion continue sur la langue, laquelle, comme le souligne Lusetti (2008, p. 181), «peut être le moyen de développer les compétences épilinguistiques ..., qui précèdent les compétences métalinguistiques », exigées à l'école. Ces compétences métalinguistiques, qui permettent un niveau de réflexion explicite et intentionnel dirigeant des ressources attentionnelles vers des éléments linguistiques au-delà du message à transmettre (Pinto et El Euch, 2015), seraient par ailleurs un facteur influant sur le développement de la compétence langagière dans les diverses langues apprises par l'élève (Jessner, 2008).

Dans le cadre du présent texte, en harmonie avec la thématique du translanguaging que propose le présent numéro des Cahiers de l'ILOB, nous nous 
focaliserons sur cette dernière finalité du recours au réseau littéraire grammatical et nous verrons comment il peut être mis en œuvre en salle de classe de français (langue d'enseignement ou seconde) pour amener l'élève à réfléchir, en concomitance, sur la grammaire de plusieurs langues. Nous nous intéressons donc principalement au translanguaging pédagogique, c'est-à-dire aux pratiques enseignantes permettant le recours à toutes les ressources du répertoire langagier de chaque élève lors des activités de la classe (Velasco et García, 2014), particulièrement lors de l'enseignement grammatical. En ce sens, nous nous inscrivons dans le paradigme de la didactique intégrée des langues (Roulet, 1980), «une approche globale qui sous-tend de manière cohérente l'enseignement de toutes les langues» (Wokusch, 2008a, p. 30); nous avançons ainsi que le réseau littéraire peut servir de «catalyst for dual language awareness » (Lyster, 2014, p. 64), un facteur contribuant au développement d'une compétence plurilingue (Lüdi et Py, 2009; Moore, 2001). L'exploitation de ce médium, argüons-nous, contribuera notamment à la désacralisation de la leçon de grammaire à l'école, celle-ci pouvant désormais proposer un espace dans lequel les langues se côtoient et dont l'apprentissage se nourrit mutuellement.

\section{L'enseignement grammatical par l'entremise de la littérature de jeunesse en contexte plurilingue}

Les propositions que nous mettons en avant ici s'inscrivent dans un courant de recherche récent, lequel a principalement pour objectif de reconnaitre le potentiel didactique que revêt la littérature de jeunesse en contexte plurilingue et pluriculturel (Hélot, Sneddon et Daly, 2014; Moore et Sabatier, 2014). Dans cette perspective, comme le souligne Montésinos-Gelet (2012), l'utilisation réfléchie d'une littérature de qualité en contexte plurilingue et pluriculturel peut soutenir la légitimation de l'habitus linguistique et culturel de l'élève. Fleuret et Sabatier (2019) précisent quant à elles que le recours à ce médium en classe peut construire un rapport multidimensionnel au monde et actualiser, dans des contextes de diversité linguistique et culturelle, la construction des compétences en littératie.

À notre connaissance, peu de travaux abordent la littérature de jeunesse et son recours en contexte plurilingue afin de soutenir le développement de la compétence grammaticale des élèves. De manière générale, on reconnait que l'album, s'il reflète leur répertoire linguistique composite, peut promouvoir les langues qu'ils connaissent, les mettre à profit et, au regard des travaux phares de Cummins (1979) sur l'interdépendance linguistique, leur permettre de s'approcher progressivement de la langue de l'école (Armand, Gosselin-Lavoie et Combes, 2016; Cummins, 2014). Il peut également favoriser l'émergence d'un bi/plurilinguisme équilibré, car l'apprenant, reconnu et apprécié pour la pluralité de son identité linguistique, se permet constamment d'opérer des va- 
et-vient entre les langues qui composent son répertoire, ce que préconisent les tenants de la perspective éducative du translanguaging (Corcoll, 2013; Creese et Blackledge, 2010; Velasco et García, 2014).

De nature théorique, le présent texte veut, d'une part, mettre au jour des pistes pour décloisonner l'enseignement grammatical à l'aide de la littérature de jeunesse et offrir, de l'autre, des avenues à emprunter pour faire usage de réseaux littéraires grammaticaux dans une perspective plurilingue. Nous soutiendrons que, grâce à la mise en œuvre de tels réseaux, l'enseignement grammatical peut être désacralisé, accessible, réaliste et, parce qu'il est le reflet éminent des trajectoires linguistiques qui caractérisent les élèves des salles de classe aujourd'hui (Moore, 2006), il peut favoriser le développement d'une posture réflexive à l'endroit des langues mises en interaction et l'émergence d'une compétence plurilingue fonctionnelle.

\section{Des exemples de réseaux littéraires grammaticaux pour favoriser la réflexion sur les langues et valoriser le plurilinguisme}

Nous présentons dans cette section trois types de réseaux littéraires par l'intermédiaire desquels peut s'opérer l'enseignement grammatical plurilingue. Nous exemplifierons donc chaque modalité de réseau à l'aide d'œuvres et d'activités qui peuvent être réalisées afin de faire comprendre, dans plusieurs langues, les mécanismes qui régissent le fonctionnement d'un concept grammatical particulier. Notons d'ores et déjà que les œuvres sélectionnées pour les réseaux présentés ne sont pas les seules qui pourraient être utilisées pour enseigner les concepts grammaticaux retenus; d'autres titres pourraient être tout à fait appropriés pour les travailler. De manière générale, il s'agit en fait de tenir compte du potentiel grammatical que peuvent receler des œuvres de littérature de jeunesse utilisées en classe ou trouvées en bibliothèque.

\section{Le réseau littéraire grammatical composé d'une ou de plusieurs œuvres plurilingues}

La recherche commence à s'intéresser aux effets de l'utilisation des livres plurilingues (des œuvres dans lesquelles on trouve plusieurs langues) sur l'apprentissage des langues qui y sont représentées. En sus de normaliser la diversité linguistique dans un médium qui est souvent associé à une tradition régie par des normes monolingues - la littérature (Moore et Sabatier, 2014) ces livres faciliteraient notamment l'apprentissage de l'association graphèmesphonèmes (Naqvi, Thorne, Pfitscher, Nordstokke et McKeough, 2012) et le développement lexical dans les langues de l'ouvrage (Gosselin-Lavoie, 2016). D'après Sneddon (2009), en effet, la lecture de livres plurilingues « enables children to hypothesise about language and reveals what they understand about the relationship between their languages» (p. 148). 
Le réseau que nous avons élaboré permet l'enseignement de la phrase syntaxique au premier cycle de l'école élémentaire. Rappelons que la phrase syntaxique, constituée d'un sujet, d'un prédicat et de potentiels compléments de phrase, est le pilier de l'analyse dans le paradigme didactique qui fait actuellement école eu égard à l'enseignement grammatical en français au Canada (Chartrand, Aubin, Blain et Simard, 2011). Son enseignement, ipso facto, devrait débuter dès le commencement de la scolarité obligatoire en français langue d'enseignement (Lefrançois, Montésinos-Gelet et Anctil, 2018) et dès le niveau débutant en classe de langue seconde (Jean, 2017).

Le premier livre du réseau se nomme Cris d'animaux : de Paris à Pékin (Massin et Rabier, 2011). Sur chacune des pages qui le composent, on trouve une phrase syntaxique constituée d'un groupe nominal (réalisé par un déterminant et un nom) en fonction sujet et d'un prédicat formé d'un verbe intransitif. Le groupe nominal en position sujet présente toujours un animal, alors que le verbe dans le prédicat exprime le cri qu'émet cet animal (p. ex., Le chien jappe, Le loup hurle). Sont ensuite présentées les onomatopées associées au cri de l'animal dans différentes langues, ce qui octroie au livre un caractère plurilingue et pluriculturel qui peut être exploité en salle de classe.

Pour éviter d'instrumentaliser l'ouvrage, afin que l'élève puisse donc développer le gout de lire et qu'il n'associe pas automatiquement le livre au travail grammatical, nous proposons d'abord d'en explorer le contenu pour ses qualités littéraires et d'aborder, en amont des activités linguistiques, les aspects qui sont susceptibles de générer des discussions sur la diversité linguistique. Par exemple, il serait intéressant de demander aux élèves connaissant d'autres langues de prononcer le cri de chaque animal du livre, ou encore de leur faire écouter ces cris d'animaux dans différentes langues grâce à une recherche sur Internet. Quand les élèves se sont approprié le contenu du livre, par exemple lors d'une deuxième lecture de l'œuvre, on peut ouvrir une discussion sur la structure répétée qu'adoptent les phrases et en analyser, en groupe-classe, le premier énoncé : Le coq chante.

Pour activer les connaissances que peuvent détenir certains élèves concernant la phrase, l'enseignant leur pose des questions telles que «D'après vous, est-ce une phrase complète ?», «Pourquoi est-ce une phrase complète? » et «Qu'est-ce qu'il faut pour qu'une phrase soit complète?». Pour trancher les litiges lors de l'échange, il leur remet alors un tableau de classification qui engagera les élèves dans une première réflexion sur les constituants obligatoires de la phrase en français (tableau 1).

En complétant le tableau 1, en dyade ou individuellement, les élèves opèrent des analyses phrastiques et se questionnent quant aux constituants que l'on trouve dans les phrases du livre. Lors du retour en plénière, l'enseignant génère une discussion sur les observations suscitées par le travail grammati- 


\section{TABLEAU 1}

Tableau de classification adapté de Thibeault et Quevillon Lacasse (2018)

\begin{tabular}{lcc}
\hline \hline Phrases tirées du livre & De qui parle-t-on? & Qu'est-ce qu'on en dit? \\
\hline Le coq chante. & Le coq & chante. \\
La poule glousse et caquette. & & \\
La vache meugle. & & \\
\hline \hline
\end{tabular}

cal ; les élèves découvriront officiellement que dans chaque phrase de ce livre, le premier groupe de mots exprime ce dont on parle, alors que l'autre groupe de mots expose ce que l'on en dit. Ainsi, dans cette activité, on procède d'abord à une analyse des phrases à partir du sens qu'elles véhiculent, le sens étant souvent plus accessible, plus concret, pour des élèves du premier cycle (Lefrançois, Montésinos-Gelet et Anctil, 2016). Quand les élèves ont conceptualisé les caractéristiques sémantiques de la phrase, on peut nommer les concepts syntaxiques qui leur sont associés, sujet et prédicat ${ }^{1}$, et commencer un référentiel de classe sur la construction de phrases.

Nous suggérons, afin de poursuivre l'exploration de la phrase dans une perspective plurilingue, le recours au livre La ceinture fléchée/The arrow sash/ Aienkwire atiatahna (Rivard, 2014), qui porte sur l'anthropologie du vêtement chez les Premières Nations du Canada. Dans cette œuvre, une grand-mère explique à sa petite-fille l'histoire de cette ceinture traditionnelle et lui expose la signification de ses couleurs et de ses formes. On trouve, sur chaque doublepage, une phrase respectant la structure canonique sujet-prédicat, celle-ci apparaissant en français, en anglais et en mohawk. Ce caractère plurilingue de l'ouvrage permet dès lors un travail grammatical dans plus d'une langue. Avec les élèves, on peut d'abord répéter le travail de classification qui a été proposé à partir du titre précédent. Ensuite, il conviendrait de les inviter à s'exercer en leur suggérant différents jeux. On peut, entre autres, penser à une activité d'association à la manière d'un casse-tête, activité lors de laquelle les élèves doivent jumeler les sujets que l'on trouve dans les phrases du livre à leur prédicat (voir figure 1). Ainsi pourront-ils travailler les éléments lexicaux de l'ouvrage et, sur le plan grammatical, constater plus explicitement que, en anglais comme en français, le sujet précède le prédicat, et que ni l'un ni l'autre ne peut être effacé ${ }^{2}$.

\footnotetext{
${ }^{1}$ Nous invitons le lecteur à se référer à l'article de Fisher et Nadeau (2017) sur l'utilisation du terme prédicat.

${ }^{2}$ Bien entendu, des différences syntaxiques importantes existent entre l'anglais et le français, comme la position des pronoms compléments, mais l'objet de l'activité présentée ici est de sensibiliser les élèves aux similarités entre les langues (Kellerman, 1983).
} 

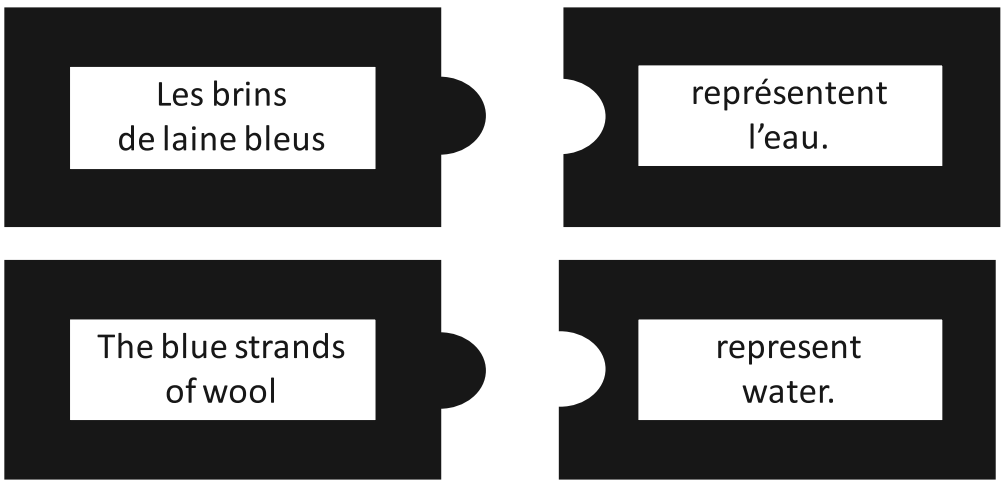

FIGURE 1

Activité d'association sur les constituants d'une phrase en français et en anglais

Il peut également être tout à fait intéressant d'inviter un locuteur du mohawk, que ce soit un élève, un parent ou un membre de la communauté, à venir lire les passages dans cette langue à la classe. Les élèves se familiariseront donc avec la langue dans son versant oral. Ce locuteur pourrait également discuter du contenu du livre avec le groupe et présenter la structure syntaxique qu'adoptent les phrases en mohawk avec les élèves. Ces derniers découvriront notamment que, contrairement à l'anglais et au français, langues qui imposent une structure canonique relativement figée, le mohawk est plus variable, car le prédicat précède souvent le sujet.

Le livre qui clôt notre premier réseau, à la différence des précédents, est une œuvre narrative; elle s'intitule Les ogres confus (École Nesbitt, 2018) et raconte l'histoire d'une ogresse francophone et d'un ogre anglophone qui tombent amoureux, mais qui ont initialement de la difficulté à communiquer en raison de leur bagage linguistique distinct. Le livre, dont certains passages sont en français, d'autres en anglais, se démarque des autres, car il est écrit et illustré par des élèves bilingues d'une école élémentaire anglophone de Montréal.

Travailler à partir d'une telle œuvre en classe peut s'avérer intéressant, et ce, pour deux raisons. D'une part, la littérature de jeunesse plurilingue demeure émergente et, en salle de classe, elle peut initialement déstabiliser les élèves qui n'ont pas l'habitude d'être exposés à des récits qui se caractérisent par la présence de codes linguistiques appartenant à des langues différentes. En utilisant un tel livre, l'enseignant montre donc aux élèves que cette littérature leur est accessible, puisqu'elle a été produite par des pairs du même âge. De l'autre, dans la foulée des travaux sur l'enseignement de l'écriture qui mettent en avant la pertinence didactique de l'étude de textes-mentors en amont de la 
production écrite par l'élève (Fletcher, 2011; Hill, 2013), l'étude de ce texte peut servir de levier afin de mettre en place des projets d'écriture novateurs intégrant des pratiques de translanguaging.

Eu égard à l'enseignement de la phrase, on remarque dès les premières pages du livre que les constructions syntaxiques adoptent pratiquement toutes la structure canonique de la phrase syntaxique en anglais et en français (sujet d'abord, prédicat ensuite). Cette transcription de la première page exemplifie ce que nous avançons :

Il était une fois deux ogres qui s'appelaient Matilda et Lucas. Matilda était une femme très princesse. Elle aimait les robes, les bagues et les fleurs. Lucas était un homme très féroce. Il aimait boxer, les souliers et les filles. Les deux s'aimaient à la folie. Matilda invited Lucas over for a date. She was nervous and excited at the same time. (École Nesbitt, 2018, p. 3)

Si les activités que nous avons présentées en lien avec les livres précédents peuvent être mises en œuvre à partir des phrases de ce livre, c'est principalement l'impact de la répétition de la seule structure sujet-prédicat sur le lecteur qui mérite ici d'être portée à l'attention des élèves. En effet, si toutes les phrases d'un texte répètent cette structure, cela peut en faciliter la compréhension, certes, mais sur le plan énonciatif, on note un certain effet saccadé, lequel peut occasionner un manque de fluidité. L'enseignant peut dès lors amener ses élèves à remarquer les impacts de cette structure répétée et, informellement, leur demander de créer des phrases plus complexes, qui pourraient contribuer à rendre la lecture du texte plus fluide. Il s'agira donc d'une occasion en or pour enseigner certains mécanismes syntaxiques, comme le complément de phrase et son déplacement en tête de phrase, et la coordination et la juxtaposition de phrases.

\section{Le réseau littéraire grammatical composé de la même đuvre dans plusieurs langues}

La deuxième modalité de réseaux littéraires grammaticaux que nous présentons renvoie à l'utilisation de plusieurs versions d'une même œuvre monolingue dans plusieurs langues. À la suite des travaux de Lyster (Lyster, 2014; Lyster, Collins et Ballinger, 2009), qui a réalisé une étude dans le cadre de laquelle des élèves en immersion française au Québec ont pris part à des activités de lecture à voix haute des mêmes livres en anglais et en français, et qui en a constaté les effets positifs sur la conscience morphologique dans les deux langues, nous avons élaboré un réseau qui adopte la modalité proposée par ce chercheur.

Pour détourner la question épineuse de l'usage de textes traduits en classe Devrait-on les utiliser ? Le livre traduit est-il légitime sur le plan littéraire ? ${ }^{3}$ -

\footnotetext{
${ }^{3}$ Pour une réflexion sur le recours à l'œuvre traduite en contexte éducatif, voir
} 
nous suggérons ici un réseau conçu à partir de l'œuvre de Marie-Louise Gay, auteure qui signe fréquemment les versions française et anglaise de ses livres. Dans ses livres Un million de questions !/Any questions? (Gay, 2014a, 2014b), elle s'engage dans un dialogue avec des élèves qui lui posent des questions sur le processus d'écriture et qui l'aident donc à construire son récit (p. ex., Do you write all day? Where does a story start?). Ce livre met donc en évidence de manière farfelue et accessible le processus d'écriture, le démystifie et permet à l'enseignant de faire des parallèles concrets entre le travail d'une auteure et celui des élèves lorsqu'ils produisent eux-mêmes des textes à l'écrit. Il peut de surcroit être la source d'un travail sur la création des questions, car le livre en comporte une quantité impressionnante. C'est l'objet grammatical sur lequel nous mettons l'accent pour exemplifier ce type de réseau.

La mise en réseau des versions anglaise et française du livre fait suite au réseau littéraire précédent, qui porte sur la phrase syntaxique. Car, rappelonsle, la phrase interrogative, en français et en anglais, se construit à partir de son homologue déclaratif. C'est donc en invitant les élèves à observer les différences syntaxiques entre les structures déclaratives et interrogatives qu'ils peuvent découvrir le fonctionnement des énoncés interrogatifs (Nadeau et Fisher, 2006).

La conception des tableaux par l'intermédiaire desquels les élèves observeront les règles qui sous-tendent la formation des questions en anglais et en français repose sur les mécanismes transformatifs — l'effacement, le déplacement, l'ajout et le remplacement - à partir desquels, dans les deux langues, la phrase conforme à la structure canonique se modifie pour adopter un autre type ou une autre forme (voir tableau 2). Même si l'élève n'aura pas à utiliser ici les quatre mécanismes syntaxiques, nous suggérons ce tableau puisqu'il peut par la suite, à titre d'outil d'analyse polyvalent, être employé à nouveau pour analyser des phrases d'un autre type ou d'une autre forme. En effet, dans les deux langues ciblées, ce sont les quatre mécanismes qui sont répertoriés lors de la transformation de phrases. Quand les élèves ont saisi les règles régissant la construction de différentes constructions interrogatives (totales, partielles, débutant par différents adverbes ou pronoms interrogatifs, etc.), on peut aisément les inviter à redécouvrir une œuvre qu'ils ont déjà lue et, à la manière de Gay dans ses livres, à s'adresser à l'auteure en lui posant des questions, à l'oral ou à l'écrit, en anglais et en français. On peut aussi envisager une situation didactique lors de laquelle les élèves doivent réagir à l'égard d'un texte écrit par un camarade, et ce, en lui posant une série de questions qui respectent la construction syntaxique de celles qui ont fait l'objet d'une observation au préalable, tout en leur laissant le choix de pratiquer le translanguaging.

Gonzáles Davies (2014). 


\section{TABLEAU 2}

Tableaux de classification adaptés de Larouche, Thibeault, Dias, Laroche, Quesnel et Valois (2016)

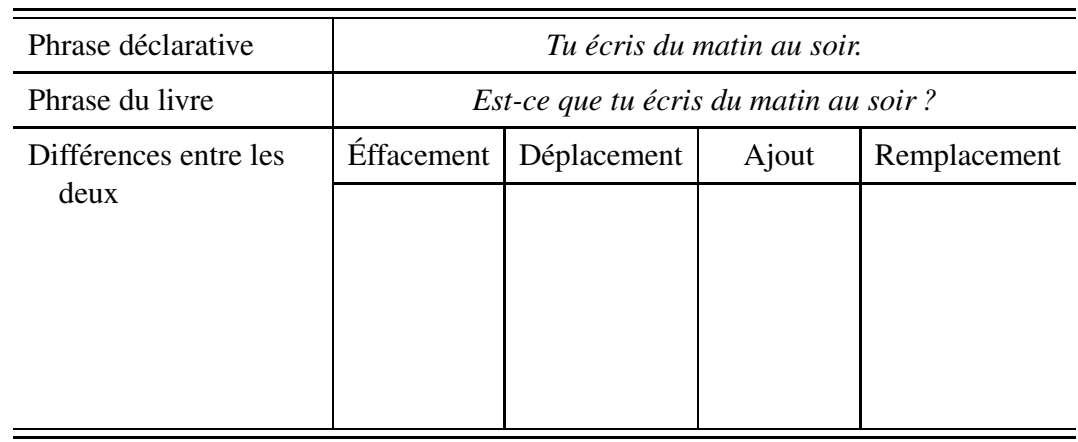

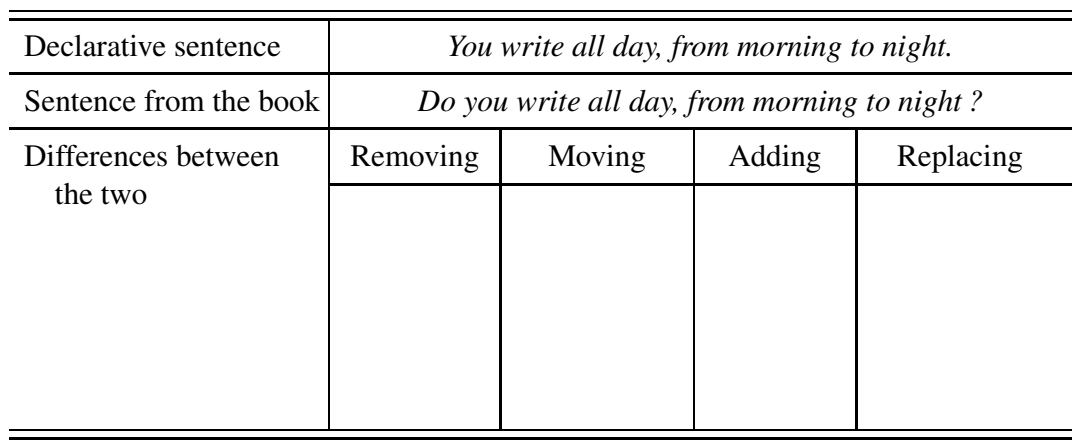

\section{Le réseau littéraire composé d'œuvres monolingues dans différentes langues}

La troisième et dernière modalité de réseau que nous suggérons dans le cadre de ce texte prend appui sur l'utilisation de titres rédigés dans différentes langues, ceux-ci renfermant des concepts linguistiques similaires d'une langue à l'autre. Ainsi, en réunissant ces œuvres dans un même réseau, l'enseignant peut faire remarquer les similitudes et les différences qui sont afférentes à un même concept dans plusieurs langues (Forel, 2014) en les contextualisant à partir de contextes littéraires qui s'insèrent dans la culture associée à la langue originale de l'ouvrage.

Le réseau que nous avons créé pour rendre compte de ce type repose sur l'enseignement de l'hypothèse en français et en anglais. Dans les deux langues, il existe plusieurs structures syntaxiques permettant d'exprimer une considération hypothétique (Vairel, 1982). En français, par exemple, le locuteur peut 
opter, entre autres, pour la construction $S i+$ verbe au présent de l'indicatif, verbe au futur simple de l'indicatif (p. ex., S'il veut venir, il viendra), la construction $S i+$ verbe au présent de l'indicatif, verbe au présent de l'impératif (p. ex., Si tu veux venir, viens!) ou encore la construction $S i+$ verbe à l'imparfait de l'indicatif, verbe au conditionnel présent (p. ex., Si tu voulais venir, tu viendrais).

En anglais comme en français, de nombreux auteurs font des usages tout à fait originaux de structures hypothétiques, structures vers lesquelles on peut attirer l'attention de l'élève. Dans le réseau que nous avons conçu, l'élève est appelé à découvrir les rouages de la structure composée de l'imparfait de l'indicatif et du conditionnel présent, et de son équivalent le plus proche en anglais. Pour le livre en langue française, nous retenons 20 bonnes raisons d'aller à l'école (Escoffier et Guyard, 2012), lequel fait entrer le lecteur dans l'imaginaire d'un enfant qui souhaite se départir de son rôle d'élève et qui se demande donc ce qu'il lui arriverait s'il pouvait cesser d'aller à l'école. Sur chaque double-page, on trouve une phrase qui adopte la structure hypothétique retenue et qui présente la conséquence de l'hypothèse précédente :

(1) Si je n'allais pas à l'école, je ne saurais ni lire, ni écrire.

(2) Si je ne savais pas lire, je confondrais les étiquettes des flacons de shampoing et des bouteilles de soda.

(3) Si j'avalais le contenu du flacon de shampoing, je ferais des bulles en parlant.

Abordant également la thématique de l'imaginaire enfantin, le livre de langue anglaise If kids ruled the world (Bailey, 2014) raconte ce que deviendrait le monde si c'étaient les enfants qui régnaient sur lui. À l'instar de l'ouvrage en français avec lequel nous le mettons en réseau, cette œuvre renferme une variété de phrases exprimant une hypothèse. Les élèves, en lisant l'une et l'autre de ces œuvres, se situeront donc dans des univers littéraires relativement analogues, ce qui favorisera sans conteste leur mise en lien thématique, d'une part, et grammaticale, de l'autre.

Afin de les amener à opérer une observation en synchronie des mécanismes structuraux qui gouvernent la création des hypothèses, l'enseignant peut extraire quelques-unes des phrases des livres et les insérer dans un tableau de classification (tableau 3), ce dernier orientant la réflexion linguistique qui permettra des questionnements ciblés et, in fine, la mise au jour de la structure en anglais et en français.

Comme on le constate, plusieurs connaissances sont préalables à la réalisation de cette activité de conscientisation. Primo, les élèves doivent être à même de repérer le verbe conjugué dans une phrase. Deuxio, ils doivent connaitre les formes qu'adoptent ces verbes dans les structures étudiées, afin de pouvoir en reconnaitre le mode-temps et, par la suite, construire à leur tour 
TABLEAU 3

Tableaux de classification pour l'observation comparée des structures hypothétiques

\begin{tabular}{l|l|l|l|l}
\hline \hline $\begin{array}{l}\text { Phrases du livre } \\
\text { en français }\end{array}$ & $\begin{array}{c}\text { Quel est le } \\
\text { premier } \\
\text { verbe? }\end{array}$ & $\begin{array}{c}\text { Quel est le } \\
\text { mode-temps de } \\
\text { ce verbe? }\end{array}$ & $\begin{array}{c}\text { Quel est le } \\
\text { deuxième } \\
\text { verbe? }\end{array}$ & $\begin{array}{c}\text { Quel est le mode- } \\
\text { temps de ce } \\
\text { deuxième verbe? }\end{array}$ \\
\hline $\begin{array}{l}\text { Si je portais des } \\
\text { couches, les } \\
\text { limaces géantes se } \\
\text { moqueraient de } \\
\text { moi. }\end{array}$ & & & & \\
\hline $\begin{array}{l}\text { S'il y avait } \\
\text { beaucoup de vent, } \\
\text { je rencontrerais } \\
\text { plein d'autres } \\
\text { enfants comme moi. }\end{array}$ & & & & \\
\hline $\begin{array}{l}\text { Si je n'allais pas à } \\
\text { l'école, je ne } \\
\text { saurais ni lire, ni } \\
\text { écrire. }\end{array}$ & & & & \\
\hline \hline
\end{tabular}

\begin{tabular}{l|l|l|l|l}
\hline \hline $\begin{array}{l}\text { Sentences from the } \\
\text { book in English }\end{array}$ & $\begin{array}{c}\text { What's the } \\
\text { first verb? }\end{array}$ & $\begin{array}{c}\text { What's the } \\
\text { verb's tense? }\end{array}$ & $\begin{array}{c}\text { What's the } \\
\text { second verb? }\end{array}$ & $\begin{array}{c}\text { What's the } \\
\text { verb's tense? }\end{array}$ \\
\hline $\begin{array}{l}\text { If kids ruled the } \\
\text { world, every day } \\
\text { would be your } \\
\text { birthday. }\end{array}$ & & & & \\
\hline $\begin{array}{l}\text { If kids ruled the } \\
\text { world, the monsters } \\
\text { in your closet } \\
\text { would be scared of } \\
\text { you! }\end{array}$ & & & & \\
\hline $\begin{array}{l}\text { If kids ruled the } \\
\text { world, all the } \\
\text { sidewalks would be } \\
\text { trampolines. }\end{array}$ & & & & \\
\hline \hline
\end{tabular}

des énoncés à valeur hypothétique. Quand les élèves ont complété chacun des deux tableaux, on peut faire un retour en plénière et formaliser avec eux, en les encourageant à comparer les modes-temps qui sont privilégiés par l'anglais et le français, les règles qui sous-tendent l'usage des structures hypothétiques 
étudiées. Ils pourront également noter la présence d'une virgule dans les deux langues, ce signe de ponctuation indiquant le détachement du complément de phrase et sa présence en tête de phrase.

\section{Conclusion et pistes de recherche futures}

Dans un article relevant les principes qui sous-tendent la didactique intégrée des langues, Wokusch (2008b) mentionne l'importance de recourir «à des démarches compatibles dans toutes les langues pour aborder des aspects techniques (vocabulaire, grammaire, textes) ( (p. 13). Elle précise en outre que l'analyse inductive d'un phénomène grammatical pour inférer les règles qui en régissent le fonctionnement renvoie à une piste prometteuse et que, grâce à elle, l'apprenant peut mettre en relation les contenus qu'on lui enseigne, les distinguer et, en bout de ligne, les utiliser à dessein d'une langue à l'autre.

C'est, en définitive, l'exemple d'une telle démarche que nous avons voulu mettre en avant dans le cadre de cet article. Nous avons avancé que la littérature de jeunesse peut exposer et sensibiliser l'élève à la diversité linguistique et culturelle (Fleuret et Sabatier, 2019; Montésinos-Gelet, 2012), mais qu'à l'aide d'une démarche didactique qui repose sur l'analyse des choix linguistiques qu'opèrent les auteurs, l'enseignant peut aussi l'amener à adopter une posture réflexive vis-à-vis des phénomènes à l'étude dans plusieurs langues. Ce faisant, l'enseignement de la grammaire que l'on propose délaisse les fondements monolingues à partir desquels il s'édifie depuis longtemps et prépare l'apprenant à évoluer dans un monde marqué par un plurilinguisme indéniable.

Les propositions didactiques exposées ici n'ont pas encore fait l'objet de recherches empiriques. En effet, nous publions divers exemples de réseaux littéraires grammaticaux dans des réseaux professionnels de l'enseignement depuis quelques années déjà, dans le but de recueillir des commentaires de la part des enseignants et d'éventuellement ajuster nos dispositifs. Nous envisageons, dans un futur proche, de vérifier l'effet de telles pratiques d'enseignement dans des classes de français langue d'enseignement et de langue seconde sur différents aspects de l'apprentissage de la grammaire.

\section{Références}

Adair-Hauck, B. et Donato, R. (2002). The PACE model : A story-based approach to meaning and form for standards-based language learning. The French Review, 76, 265-275.

Armand, F., Gosselin-Lavoie, C. et Combes, É. (2016). Littérature jeunesse, éducation inclusive et approches plurielles des langues. Synergies Canada, 9, 1-5.

Bailey, L. (2014). If kids ruled the world. Toronto : Kids Can Press.

Beaulne, G. et Gauvin, I. (2017). Effets d'un enseignement suivant la méthode de la médiation sociocognitive des apprentissages sur la performance d'élèves de première 
secondaire à identifier le sujet. Language and Literacy, 19(14), 1-22.

Blain, S., Cavanagh, M. et Cammarata, L. (2018). Enseigner l'écriture en milieu francophone minoritaire : comment motiver les élèves et surmonter les défis de l'insécurité linguistique ? Revue canadienne de l'éducation, 41(4), 1105-1131.

Chartrand, S.-G. (1996). Apprendre la grammaire par la démarche active de découverte. Dans S.-G. Chartrand (dir.), Pour un nouvel enseignement de la grammaire (2e éd., p. 197-226). Montréal : Les Éditions Logiques.

Chartrand, S.-G. (2012). Quelles finalités pour l'enseignement grammatical à l'école ? Une analyse des points de vue de didacticiens du français depuis 25 ans. Formation et profession, 20(3), 48-59.

Chartrand, S.-G. (2016). Donner un second souffle à la rénovation de l'enseignement grammatical. Dans S.-G. Chartrand (dir.), Mieux enseigner la grammaire : pistes didactiques et activités pour la classe (p. 1-4). Montréal : Éditions du renouveau pédagogique (ÉRPI).

Chartrand, S.-G., Aubin, D., Blain, R. et Simard, C. (2011). Grammaire pédagogique du français d'aujourd'hui (2e éd.). Montréal : Chenelière Éducation.

Chartrand, S.-G. et Boivin, M.-C. (2004). Articulation des activités métalinguistiques aux activités discursives dans la classe de français au secondaire inférieur. Dans É. Falardeau, C. Fisher, C. Simard et N. Sorin (dir.), Le français : discipline singulière, plurielle ou transversale? (p. 1-20) [Actes du 9e colloque de l'Association internationale pour la recherche en didactique du français (AiRDF)]. Québec : AiRDF. www.enseignementdufrancais.fse.ulaval.ca/fichiers/site_ens_francais/modules/ document_section_fichier/fichier_ee3628e1eaf3_grammaire_lecture_et_ecriture.pdf

Chénard-Guay, C. (2011). Écrire à la manière d'un auteur au premier cycle du primaire : pratiques et impacts (Mémoire de maitrise inédit). Université de Montréal, Montréal. papyrus.bib.umontreal.ca/xmlui/handle/1866/4935

Chervel, A. (2006). Histoire de l'enseignement du français du XVIIe au XXe siècle. Paris : Retz.

Corcoll, C. (2013). Developing children's language awareness : switching codes in the language classroom. International Journal of Multilingualism, 10, 27-45.

Creese, A. et Blackledge, A. (2010). Translanguaging in the bilingual classroom : A pedagogy for learning and teaching ? Modern Language Journal, 94, 103-115.

Cummins, J. (1979). Cognitive/academic language proficiency, linguistic interdependance, the optimum age question and some other matters. Working Papers on Bilingualism, 19, 121-129.

Cummins, J. (2014). Foreword. Dans C. Hélot, R. Sneddon et N. Daly (dir.), Children's literature in the multilingual classroom (p. 1-7). Londres : Trentham Books.

De Pietro, J.-F. (2006). Entre grammaire utile et ouverture à la diversité des langues, quelles perspectives pour des activités métalangagières à l'école ? Langage et pratiques, 38, 7-23. 
Dupin de Saint-André, M., Montésinos-Gelet, I. et Bourdeau, R. (2015). Intégrer la littérature jeunesse en classe à l'aide de réseaux littéraires. Documentation et bibliothèques, 61, 22-31.

École Nesbitt. (2018). Les ogres confus. Montréal : Une école montréalaise pour tous.

Ellis, R. et Shintani, N. (2014). Exploring language pedagogy through second language acquisition research. Milton Park, Angleterre : Routledge Taylor \& Francis.

Escoffier, M. et Guyard, R. (2012). 20 bonnes raisons d'aller à l'école. Paris : Éditions Frimousse.

Fisher, C. et Nadeau, M. (2017). Survivre au prédicat : le cas du Québec. Pratiques : linguistique, littérature, didactique, 175-176, 1-18.

Fletcher, R. (2011). Mentor author, mentor texts : Short texts, craft notes, and practical classroom uses. Portsmouth, NJ : Heinemann.

Fleuret, C. et Sabatier, C. (2019). La littérature de jeunesse en contextes plurilingues et pluriculturels : quels enjeux didactiques pour quelles pratiques d'enseignementapprentissage et pour quelles perspectives interculturelles? Recherches et applications, 65, 95-112.

Forel, C. (2014). Pour une approche plurielle de la grammaire. Babylonia, 2, 72-76.

Gay, M.-L. (2014a). Any questions? Saint-Lambert, QC : Dominique et compagnie.

Gay, M.-L. (2014b). Un million de questions! Saint-Lambert, QC : Dominique et compagnie.

Gonzáles Davies, M. (2014). The changing role of translators in a bilingual context : Catalan (in)visibility and the translation of children's literature. Dans C. Hélot, R. Sneddon et N. Daly (dir.), Children's literature in the multilingual classroom (p. 18-34). Londres : Trentham Books.

Gosselin-Lavoie, C. (2016). Lecture de livres bilingues par six duos parent-enfant allophones du préscolaire : description des lectures et des interactions et relations avec l'acquisition du vocabulaire (Mémoire de maitrise inédit). Université de Montréal, Montréal. papyrus.bib.umontreal.ca/xmlui/bitstream/handle/1866/16439/ Gosselin-Lavoie_Catherine_memoire_2016.pdf?sequence=4\&isAllowed=y

Halliday, M.A.K. et Matthiessen, C.M.I.M. (2014). Halliday's introduction to functional grammar (4e éd.). New York : Routledge.

Hélot, C., Sneddon, R. et Daly, N. (dir.). (2014). Children's literature for the multilingual classrooms. Londres: Trentham Books.

Hill, D. (2013). Three mentor texts that support code-switching pedagogies. Voices from the middle [numéro thématique : Teaching the language of school and academics], 20(4), 10-15.

Hirvela, A. (2016). Connecting reading \& writing in second language writing instruction (2e éd.). Ann Arbour, MI : University of Michigan Press. 
Jean, G. (2017). Le point sur la grammaire «nouvelle» en FL2. Les Reflets du 35e congrès de l'AQEFLS [1'Association québécoise des enseignants de français langue seconde (AQEFLS)], 34, 20-30.

Jean, G. et Simard, D. (2011). Grammar teaching and learning in L2 : Necessary, but boring ? Foreign Language Annals, 44, 467-494.

Jessner, U. (2008). A DST model of multilingualism and the role of metalinguistic awareness. Modern Language Journal, 92, 270-283.

Katz, S.L. et Blyth, C.S. (2007). Teaching French grammar in context : Theory and practice. New Haven, CT : Yale University Press.

Kellerman, E. (1983). Now you see it, now you don't. Dans S. Gass et L. Selinker (dir.), Language transfer in language learning (p. 112-134). Rowley, MA : Newbury House.

Larouche, L. (2018). Pratiques déclarées d'enseignants de l'intermédiaire en contexte minoritaire, plurilingue et pluriethnique relativement à l'enseignement de la grammaire (Mémoire de maitrise inédit). Université d'Ottawa, Ottawa.

Larouche, L., Thibeault, J., Dias, D., Laroche, C., Quesnel, S. et Valois, M. (2016). La grammaire nouvelle : approches pédagogiques pour bien accompagner dans son apprentissage du français en milieu plurilingue et minoritaire. Ottawa : Éditions du Centre franco-ontarien de ressources pédagogiques (CFORP).

Lefrançois, P., Montésinos-Gelet, I. et Anctil, D. (2016). La conception de la phrase chez les enseignants et les élèves québécois du primaire. Lidil. Revue de linguistique et de didactique des langues, 54, 75-91.

Lefrançois, P., Montésinos-Gelet, I. et Anctil, D. (2018). Enseigner la phrase par la littérature jeunesse : fondements et activités pour soutenir la compétence à écrire au primaire. Montréal : Chenelière Éducation.

Lord, M.-A. (2012). L'enseignement grammatical au secondaire québécois : pratiques et représentations d'enseignants de français (Thèse de doctorat inédit). Université Laval, Laval. corpus.ulaval.ca/jspui/handle/20.500.11794/23457

Lüdi, G. et Py, B. (2009). To be or not to be ... a plurilingual speaker. International Journal of Multilingualism, 6, 154-167.

Lusetti, M. (2008). La langue en jeu(x) dans la littérature de jeunesse : entre enseignement de la grammaire et de la littérature. Recherches, 48, 165-187.

Lyster, R. (2014). Children's literature as a catalyst for dual language awareness. Dans C. Hélot, R. Sneddon et N. Daly (dir.), Children's literature in the multilingual classroom (p. 64-83). Londres : Trentham Books.

Lyster, R., Collins, L. et Ballinger, S. (2009). Linking languages through a bilingual read-aloud project. Language Awareness, 18, 366-383.

Marmy Cusin, V. et Schneuwly, B. (2013). Intégrer des apprentissages grammaticaux à l'étude d'un genre textuel : entre les dires et les faires. Dans O. Bertrand et 
I. Schaffner (dir.), Enseigner la grammaire (p. 357-376). Paris : Éditions de l'École polytechnique.

Massin et Rabier, B. (2011). Cris d'animaux : de Paris à Pékin. Paris : Calligram.

Montésinos-Gelet (2012). Partir d'œuvres de littérature de jeunesse pour explorer le capital culturel des élèves. In C. Fleuret et I. Montesinos-Gelet (dir.), Le rapport à l'écrit : habitus culturel et diversité (p. 9-19). Québec : Presses de l'Université du Québec.

Montésinos-Gelet, I. (2016). Description de dispositifs d'enseignement : le jogging d'écriture. Le Pollen, 20, 142-154.

Moore, D. (2001). Une didactique de l'alternance pour mieux apprendre ? ELA. Études de linguistique appliquée, 121, 71-78.

Moore, D. (2006). Plurilinguismes et école. Paris : Didier.

Moore, D. et Sabatier, C. (2014). Les approches plurielles et les livres plurilingues. De nouvelles ouvertures pour l'entrée dans l'écrit et pour favoriser le lien famille-école en milieu multilingue et multiculturel. Nouveaux cahiers de la recherche en éducation [numéro thématique : Lire et écrire : les liens école-familles-communautés en contextes pluriels], 17(2), 32-65.

Nadeau, M. et Fisher, C. (2006). La grammaire nouvelle. La comprendre et l'enseigner. Montréal : Gaëtan Morin.

Naqvi, R., Thorne, K.J., Pfitscher, C.M., Nordstokke, D.W. et McKeough, A. (2012). Reading dual language books : Improving early literacy skills in linguistically diverse classrooms. Journal of Early Childhood Research, 11, 3-15.

Pinto, A. et El Euch, S. (2015). La conscience métalinguistique : Théorie, développement et instruments de mesure. Québec : Presses de l'Université Laval.

Rivard, S. (2014). La ceinture fléchée/The arrow sash/Aienkwire atiatahna. Wendake, QC : Hannenorak.

Roulet, E. (1980). Langue maternelle et langues secondes : vers une pédagogie intégrée. Paris : Hatier et le Centre de recherche et d'étude pour la diffusion du français (CREDIF).

Smith, V. (2016). Children's literature in the classroom and curriculum. Dans D. Wyse, L. Hayward et J. Pandya (dir.), The SAGE handbook of curriculum, pedagogy, and assessment (p. 606-620). Thousand Oak, CA : Sage.

Sneddon, R. (2009). Bilingual books - Biliterate children : Learning to read through dual-language books. Londres : Trentham Books.

Tauveron, C. (2002). Lire la littérature à l'école : pourquoi et comment conduire cet apprentissage spécifique? De la GC au CM. Paris : Hatier.

Thibeault, J. et Quevillon Lacasse, C. (2016a). Il était une fois la grammaire : une chronique sur l'élaboration de réseaux littéraires pour décloisonner l'enseignement grammatical. Le Pollen, 19, 82-90. 
Thibeault, J. et Quevillon Lacasse, C. (2016b). Enseigner la grammaire de façon progressive grâce à la littérature de jeunesse : l'exemple du complément de phrase. Le Pollen, 21, 134-142.

Thibeault, J. et Quevillon Lacasse, C. (2018). Commencer l'enseignement grammatical du bon pied : la phrase syntaxique et son enseignement dès la première année. Le Pollen, 25, 134-144.

Vairel, H. (1982). Les phrases conditionnelles/hypothétiques en français : la valeur de si A, B. L'information grammaticale, 14, 5-10.

Vargas, C. (1996). Grammaire et didactique plurinormaliste du français. Repères, 14, 83-103.

Velasco, P. et García, O. (2014). Translanguaging and the writing of bilingual learners. Bilingual Research Journal, 37, 6-23. doi.org/10.1080/15235882.2014.893270

Wokusch, S. (2008a). Didactique intégrée des langues : la contribution de l'e ?cole au plurilinguisme des éléves. Babylonia, 1(8), 12-14.

Wokusch, S. (2008b). Didactique intégrée des langues (étrangères) à l'école : vers l'enseignement des langues de demain. Prismes, 8, 30-34. 